\title{
Star Identification Algorithm Based on Oriented Singular Value Feature and Reliability Evaluation Method*
}

\author{
Xin WeI, ${ }^{1,2) \dagger}$ Desheng Wen, ${ }^{1)}$ Zongxi Song,${ }^{1)}$ and Jiangbo $\mathrm{XI}^{3)}$ \\ ${ }^{1}$ Xi' an Institute of Optics and Precision Mechanics, Chinese Academy of Science, Xi'an 710119, China \\ ${ }^{2)}$ University of Chinese Academy of Sciences, Beijing 100039, China \\ ${ }^{3)}$ Chang'an University, Xi'an 710064, China
}

\begin{abstract}
A full-sky autonomous star identification algorithm aimed at solving the "lost-in-space" problem is presented in this paper. It mainly consists of two steps: an initial match step and a reliability evaluation step. Oriented singular value feature matching is adopted to search for corresponding candidates of the stars detected in the initial match. After obtaining the stars' initial match results, an evaluation method is applied to estimate the reliability of candidates from the star voting results, acquiring the final unique matching of stars in the image. Experiments show that our algorithm is more robust to star position noise and magnitude noise than the two conventional algorithms. In the simulations, our algorithm achieves an identification rate of $97.0 \%$ with 2-pixel star position noise and $0.3 \mathrm{Mv}$ star magnitude noise, and also performs well with false stars in the field of view. In addition, the memory requirement and identification time of our method are acceptable for actual engineering projects.
\end{abstract}

Key Words: Star Identification, Star Sensor, Spacecraft, Attitude Determination

\section{Introduction}

Reliable and autonomous attitude determination is important for both orbiting and interplanetary spacecraft. ${ }^{1,2)}$ Star sensors, also called star trackers, supply the most precise three-axis attitude reference data among many attitude determination devices, and are widely used in modern attitude and control systems.

For the star sensor, the critical process in realizing attitude estimation is accurate identification of stars in the field of view (FOV). This process consists of two typical working modes: lost-in-space (LIS) and tracking. Full-sky autonomous star identification ${ }^{3)}$ must be performed to establish an initial attitude without any prior information when the star sensor is first activated or when it loses attitude data. Once the initial attitude has been acquired, the star sensor switches to tracking mode. Generally, star identification in LIS mode is much more difficult than in tracking mode.

Existing star identification algorithms can be roughly categorized into two groups: pattern recognition and subgraph isomorphism algorithms.

The pattern recognition algorithms associate each star with an individual pattern or signature determined by its surrounding stars. In this case, the stars in a database having the closest patterns to those observed are considered as match results. In 1997, Padgett and Kreutz-Delgado first proposed the grid algorithm, ${ }^{4)}$ which is the most representative patternbased method. The grid algorithm performs well in terms of identification rate, efficiency and memory capacity.

However, the probability of selecting the closest neighbor-

(C) 2019 The Japan Society for Aeronautical and Space Sciences

*Received 18 March 2018; final revision received 19 October 2018; accepted for publication 8 February 2019.

Corresponding author, weixin@opt.cn ing star correctly can be quite low, particularly when there is noise. Although $\mathrm{Na}$ et al., ${ }^{5)}$ Clouse and Padgett ${ }^{6)}$ and Lee and $\mathrm{Bang}^{7)}$ have refined the grid algorithm, it still remains an issue. Some other patterns are utilized to overcome the grid algorithm's need to find a subaltern star. Italian scholar Silani and Lovera proposed the polar star algorithm based on the radial pattern. ${ }^{8)}$ However, this algorithm cannot comprehensively describe the distribution of neighboring stars. Moreover, the polar algorithm performs poorly with large position noise. An algorithm based on radial and cyclic features was presented by Zhang et al. ${ }^{9)}$ The cyclic pattern reduces ambiguous matches to some extent. Nevertheless, it is unreliable because of its sensitivity to noise. Wei et al. ${ }^{10)}$ introduced log-polar transform (LPT) into a star identification field, and the LPT algorithm was innovated in 2007. The LPT algorithm is robust to noise, but extremely time-consuming in the process of comparing feature vectors. In recent years, a completely new pattern method, the singular value decomposition (SVD) algorithm ${ }^{11)}$ was proposed, combining the star identification process with attitude estimation. In the SVD algorithm, the singular value feature is robust to uncertainty, but the brightness rank order of stars detected is easily influenced by the magnitude noise, which may cause patterns to be constructed with the wrong stars.

Subgraph isomorphism algorithms tend to approach star identification as an instance of subgraph isomorphism, which treats stars detected as vertices in a graph whose edges correspond to the angular separation among neighboring stars in the same FOV. Identification occurs when the undirected graph formed by several stars in the image obtained is uniquely identified with a portion of the onboard database. These algorithms typically employ star pairs, triples, or polygons to build the database; examples include the triangle algorithm, ${ }^{12,13)}$ pyramid algorithm, ${ }^{14)}$ and group match algo- 
rithm. ${ }^{15)}$ The conventional triangle algorithm was proposed by Liebe in 1995. Redundant matches always occur with star position noise, and massive memory capacity is required for the triangle algorithm. Mortari et al. introduced the pyramid algorithm, where the k-vector approach is used in the search phase. The pyramid algorithm is robust to false stars, but identification time increases exponentially with numerous false stars in the image plane. The group match algorithm is sensitive to noise, and efficiency of the algorithm degrades when spikes exist in the FOV.

To improve the robustness and efficiency of star identification, our algorithm utilizes the oriented singular value feature of triangles to acquire candidates for the several stars detected, and the reliability evaluation method is adopted for final matching results. Compared with the grid and SVD algorithms, our algorithm performs better for the occasion of star position and magnitude noise. It also has a higher identification rate with the presence of false stars in the FOV.

This paper is organized in the following manner. Section 2 describes our algorithm in detail, including the initial matching based on the oriented singular value feature and the reliability evaluation method. In Section 3, several simulations are conducted to evaluate our algorithm and compare its performance with the grid and SVD algorithms. In Section 4, we employ a probabilistic model to demonstrate robustness and efficiency of the algorithm proposed. Finally, conclusions are drawn in Section 5.

\section{Algorithm Description}

In this section, we provide a number of implementation details regarding our full-sky star identification algorithm, which involves two steps: oriented singular value feature matching and reliability evaluation. To improve the efficiency of the algorithm, we also describe onboard database generation and the data structure, in particular. A flowchart of our method is then presented.

\subsection{Oriented singular value generation}

The dedicated oriented SVD transformation utilized in this paper involves constructing an onboard database for all guide stars whose directions in the reference frame are already known. Candidates of stars observed are acquired by comparing the pattern feature derived from the image obtained with those stored in the database. Given a catalog star, its corresponding oriented singular value is generated in the following manner.

\subsubsection{Triangle pattern construction}

Considering a catalog star $\mathrm{S}_{1}$ as the pole star, the two brightest neighboring stars $\mathrm{S}_{2}$ and $\mathrm{S}_{3}$ that fall inside the region defined by the buffer radius $r$ and pattern radius $R$ are selected to build a triangle pattern $\triangle S_{1} S_{2} S_{3}$ with $S_{1}$, as shown in Fig. 1. In the above procedure, $R$ is restricted by the FOV size of the star sensor, and $r$ is introduced in the pattern generation to eliminate the adverse effect of close stars. Without loss of generality, the magnitude of $S_{2}$ is brighter than that of $S_{3}$ in the triangle $\triangle S_{1} S_{2} S_{3}$.

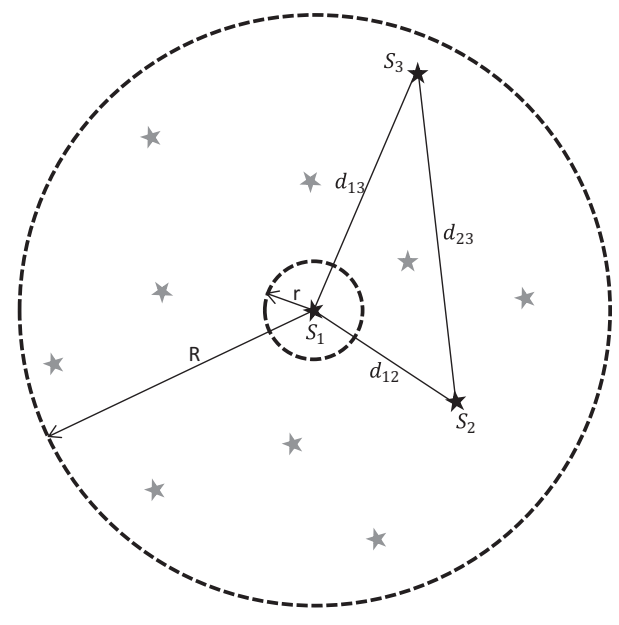

Fig. 1. Construction of the triangle pattern.

\subsubsection{Oriented SVD transformation}

An oriented coefficient is assigned to the triangle pattern $\triangle S_{1} S_{2} S_{3}$, and its value is determined by the geometric characteristics of the pattern as follows: The oriented coefficient is 1 if the rotation from vector $S_{1} S_{2}$ to vector $S_{1} S_{3}$ is done in the anti-trigonometric direction; the oriented coefficient is -1 if the rotation from vector $S_{1} S_{2}$ to vector $S_{1} S_{3}$ is done in the trigonometric direction.

After obtaining the oriented coefficient of the triangle pattern, its corresponding oriented singular value is calculated. The feature matrix of the triangle pattern $\triangle S_{1} S_{2} S_{3}$ is defined by

$$
G=\left[\begin{array}{ccc}
0 & d_{12} & d_{13} \\
d_{21} & 0 & d_{23} \\
d_{31} & d_{32} & 0
\end{array}\right]
$$

where, the element $d_{i j}$ in the matrix $G$ is the angular distance between stars $i$ and $j$ in the catalog, and can be calculated using Eq. (2):

$$
\begin{aligned}
d_{i j}= & \arccos \left(\cos \left(\delta_{i}\right) * \cos \left(\delta_{j}\right) * \cos \left(\alpha_{i}-\alpha_{j}\right)\right. \\
& \left.+\sin \left(\delta_{i}\right) * \sin \left(\delta_{j}\right)\right)
\end{aligned}
$$

where, $\alpha_{i}$ and $\delta_{i}$ are the ascension and declination of star $i$, respectively, and $\alpha_{j}$ and $\delta_{j}$ are the ascension and declination of star $j$, respectively. Then we can obtain the singular value of the feature matrix $G$ through a SVD

$$
G=U \Lambda V^{T}
$$

where, matrices $U$ and $V$ are orthogonal, and $\Lambda$ is a diagonal matrix that includes three singular values of $G$ in descending order; namely, $e_{1}, e_{2}$ and $e_{3}$. We use $c o$ when referring to the oriented coefficient of the triangle pattern, such that the oriented singular vector $P$ is given by

$$
P=\left[\begin{array}{lll}
p_{1} & p_{2} & p_{3}
\end{array}\right]=c o *\left[\begin{array}{lll}
e_{1} & e_{2} & e_{3}
\end{array}\right]
$$

The elements $p_{1}$ and $p_{2}$ in vector $P$ retain the major geometric information of the triangle, and are defined as the oriented singular values of $\triangle S_{1} S_{2} S_{3}$. By employing oriented SVD transformation, the triangle pattern of the catalog star is converted into two oriented singular values $p_{1}$ and 


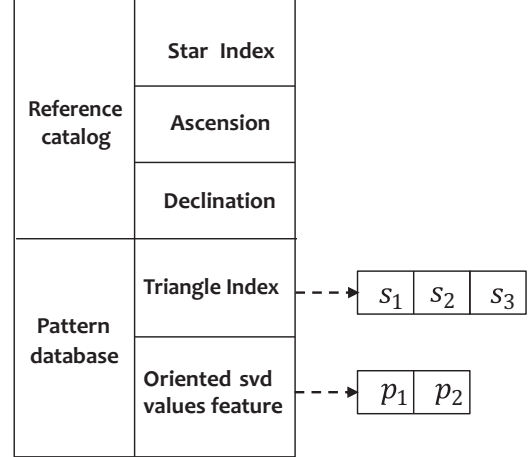

Fig. 2. Structure of the database.

$p_{2}$, which are used to obtain candidate stars in the initial match.

\subsection{Onboard database generation}

The onboard database in our algorithm consists of the reference catalog and pattern database. The structure of the database is shown in Fig. 2.

In this study, we assume a FOV of $16 \times 16^{\circ}$ and stars brighter than $6.0 \mathrm{Mv}$ can be detected by the sensor. In this case, a total of 5103 stars with apparent magnitudes down to $6.0 \mathrm{Mv}$ are selected from the SAO J2000 catalog. As double stars are close in the direction of line-of-sight, their star spots on the image sensor plane are difficult to separate from each other. To eliminate the adverse effect of double stars in the star identification procedure, we label double stars and treat them as a single synthetic star whose position is the barycenter and magnitude is the integrative brightness of the double stars. In order to reduce memory requirements for the onboard database, a uniform scan (with $0.5^{\circ}$ steps) is carried out across the entire celestial sphere and the brightest $a$ (we set $a=10$ ) stars within the FOV at each orientation are added to the reference catalog.

After selecting these guide stars from the celestial sphere, the triangle pattern is then constructed for each guide star in the reference catalog, and $p_{1}$ and $p_{2}$ are calculated using the oriented SVD transformation. To improve the efficiency of obtaining candidates, the binary search method is performed in initial matching, such that all triangle patterns in the pattern database are sorted according to ascending values of $p_{1}$.

In the database, there are 4639 guide stars in the reference catalog, and their star index (in 16 bit), ascension (in 32 bit) and declination (in 32 bit) cost about 45.3 KB (4639 $\times$ $(16+32+32)$ bits $)$ memory. For the pattern database, the triangle index unit $(3 \times 16$ bits $)$ includes three star indices of stars in the triangle pattern, and $p_{1}$ and $p_{2}$ are included in the oriented SVD values feature unit $(2 \times(32+32)$ bits $)$, such that approximately $99.7 \mathrm{~KB}$ of memory must be used to store the pattern database.

\subsection{Algorithm step}

The star identification algorithm begins by inputting the coordinates measured and apparent brightness of the stars in the imaging plane. The algorithm proposed obtains candidate stars for some of the bright stars detected by matching the oriented singular feature. Unfortunately, the oriented sin- gular value of an observed star is usually similar to several catalog stars, which brings results in redundant matches. Thus, an additional filter process must be performed to obtain unambiguous identification results.

Taking this into consideration, our star identification algorithm consists of two processes to determine the final match; namely, the initial match based on the oriented singular feature and the reliability evaluation method. The former includes oriented SVD transformation and searching for candidate stars in the database; the latter utilizes star voting to evaluate the reliability of the results obtained in the initial match and acquire unique pairings between the detected and catalog stars.

\subsubsection{Oriented singular feature match}

To minimize the risk of generating spurious matches and optimize the efficiency of the algorithm, ideally triangle patterns are constructed only for the brightest $a$ stars in the image. Considering the influence of brightness measurement error in the actual application, the brightest $c * a(c>1)$ stars detected are selected to be identified in the initial match.

By taking the brightest $c * a$ stars as sensor reference stars (SRSs), and generating a triangle pattern for each SRS by similar methods to those described in Section 2, the oriented singular feature of each triangle pattern can be extracted via the oriented SVD transformation. In the above operations, the angular distance between two detected stars A and B is calculated easily using Eq. (5)

$$
d_{\mathrm{AB}}=\arccos \left(\frac{x_{a} * x_{b}+y_{a} * y_{b}+f^{2}}{\sqrt{x_{a}^{2}+y_{a}^{2}+f^{2}} * \sqrt{x_{b}^{2}+y_{b}^{2}+f^{2}}}\right)
$$

where, $\left(x_{a}, y_{a}\right)$ and $\left(x_{b}, y_{b}\right)$ are the image coordinates of stars $\mathrm{A}$ and $\mathrm{B}$, respectively. $f$ denotes the optical length of the star sensor.

After calculating the oriented singular values $\bar{p}_{1}^{i}, \bar{p}_{2}^{i}$ $(i \in\{1,2,3 \ldots c * a\})$ for all triangle patterns in the image, we compare $\bar{p}_{1}^{i}, \bar{p}_{2}^{i}$ of the stars detected with the onboard database. Guide stars whose oriented singular values are close to those of the stars detected are considered as candidates. An example of finding the candidate stars of the jth star detected is presented to elaborate the oriented singular feature matching.

During implementation of the matching process, a fast binary search method is first applied to select the catalog stars whose oriented singular value $p_{1}$ is within $\left(\bar{p}_{1}^{j}-\varepsilon_{1}, \bar{p}_{1}^{j}+\varepsilon_{1}\right)$ from the database, and add them to set $\mathrm{K}$. The $p_{2}$ value of all elements in set $\mathrm{K}$ is tested one by one, and the guide stars whose oriented singular value $p_{2}$ is within $\left(\bar{p}_{2}^{j}-\varepsilon_{2}, \bar{p}_{2}^{j}+\varepsilon_{2}\right)$ are considered as candidates. $\varepsilon_{1}$ and $\varepsilon_{2}$ denote the permissible error of the oriented singular value $\bar{p}_{1}^{j}$, and $\bar{p}_{2}^{j}$, respectively.

\subsubsection{Reliability evaluation method}

After completing the initial matching based on the oriented singular feature, the candidates for several bright stars detected by the sensor are determined. Unfortunately, there may be some redundant matches, and even incorrect matches in these results, which can be fatal in the subsequent calculation of attitude reference data. Therefore, the reliability eval- 


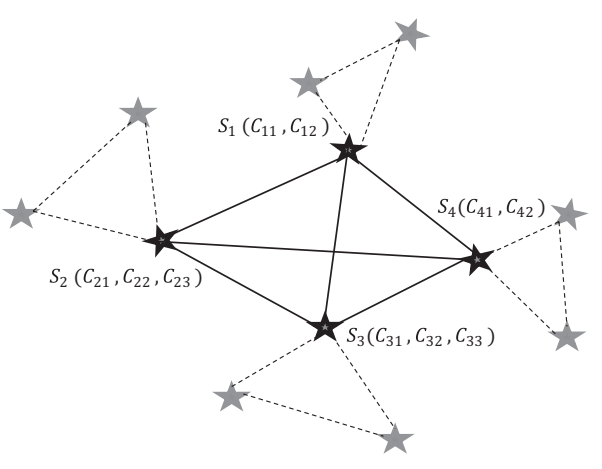

Fig. 3. Star voting.

uation method is utilized to obtain final unique matching results in our algorithm. This procedure mainly consists of three processes; namely, star voting, reliability evaluation, and validation.

Star voting process: Without the loss of generality, assume that there are four triangle patterns observed whose associated SRSs have candidates (see Fig. 3). In the illustration, $\mathrm{S}_{i}$ denotes the $i$ th SRS in the image, and $\mathrm{C}_{i j}$ denotes the $j$ th candidate star of the $i$ th SRS. For instance, the SRS $\mathrm{S}_{2}$ has three candidate stars and $S_{2}$ may correspond to the $C_{21}, C_{22}$, or $C_{23}$ guide stars in the catalog.

We first assign a set of counters to all candidate stars, where each counter is associated with a candidate. The value of the counters is set to zero at the beginning of the star voting process. Candidate stars that belong to different SRSs are selected to construct a star pairs database $D$. Subsequently, the angular distance of all star pairs detected is calculated, and $\theta_{i j}^{s}$ denotes the distance between the stars $\left(\mathrm{S}_{i}, \mathrm{~S}_{j}\right)$. For the catalog star pairs $\left(\mathrm{C}_{i m}, \mathrm{C}_{j n}\right)$ in $D, \theta_{i m, j n}^{C}$ denotes their corresponding angular distance. If the difference between $\theta_{i m, j n}^{C}$ and the distance of the corresponding stars detected $\theta_{i j}^{s}$ is less than the tolerance threshold $\varepsilon$, the star pair $\left(\mathrm{C}_{i m}, \mathrm{C}_{j n}\right)$ is considered a match with the star pair detected $\left(\mathrm{S}_{i}, \mathrm{~S}_{j}\right)$. Accordingly, $\mathrm{S}_{i}$ obtains a vote from $\mathrm{C}_{i m}, \mathrm{~S}_{j}$ obtains a vote from $\mathrm{C}_{j n}$, and the respective counters related to candidate stars $\mathrm{C}_{i m}$ and $\mathrm{C}_{j n}$ increase by one. Repeat this process until the last star pair detected has been tentatively matched.

Reliability evaluation: When the star voting process ends, the reliability of initial matching results is evaluated. A unique candidate of the star detected is defined as the corresponding candidate star of the maximum value in the counter, and $v n$ denotes the value of the counter associated with the unique candidate. For example, if the counters of candidate stars $C_{21}, C_{22}$ and $C_{23}$ are assumed to be 3,1 , and 0 after star voting, then $\mathrm{C}_{21}$ is the unique candidate for $\mathrm{S}_{2}$ and its $v n$ is 3 .

Assuming that there are $n$ stars detected $\left(\mathrm{S}_{1}, \mathrm{~S}_{2} \cdots \mathrm{S}_{n}\right)$ in an image during the reliability evaluation process, $v n(i)$ denotes the $v n$ of $S_{i}$, and the reliability level $R l$ is determined using the following equation:

$$
R l= \begin{cases}\mathrm{H} & {\left[\sum_{i=1}^{n} \mathrm{II}(v n(i) \geq 4)\right] \geq 5} \\ \mathrm{M} \quad\left[\sum_{i=1}^{n} \mathrm{II}(v n(i) \geq 3)\right]=4 \\ & \text { or }\left[\sum_{i=1}^{n} \mathrm{II}(v n(i) \geq 2)\right]=3 \\ \mathrm{~L} & {\left[\sum_{i=1}^{n} \mathrm{II}(v n(i) \geq 1)\right] \geq 2}\end{cases}
$$

In Eq. (6), $\mathrm{II}(z)$ is the indicator function $(\mathrm{II}(z)=1$ if $z$ is true, 0 otherwise). H, M and $\mathrm{L}$ denote high, medium and low reliability levels, respectively. The criteria of $\mathrm{H}$ to $\mathrm{L}$ are checked sequentially. Once the criteria are satisfied, $R l$ is determined and the reliability evaluation process terminates. In the case where none of the criteria are satisfied, the algorithm reports a failure.

When the reliability level is $\mathrm{H}$, verification is not required, and the unique candidates whose $v n$ is more than three are considered as the final unique identities of the corresponding stars detected. In all other cases, the verification process is performed.

Validation: When the reliability level is $\mathrm{M}$, the unique candidates with a counter value $v n$ equal to or greater than $T(=2$ or 3 ) are selected, and the distance between them is compared to the distance between their corresponding stars detected. If all pairs formed by these unique candidates are matched with relevant pairs of detected stars, then the unique candidates whose $v n$ are greater than or equal to $T$ are considered as the final unique identities; otherwise, our algorithm fails to identify the stars in the image.

When the reliability level is L, we select the unique candidate with a $v n$ greater than or equal to 1 . If there is a star pair formed by these unique candidates that is matched with a corresponding detected star pair, a reference star image can be generated. If several identical triangles are found in both the image obtained and reference image, as reported for the algorithm presented in Zhang et al., ${ }^{13)}$ our algorithm outputs the catalog stars in these triangles as the final unique identities of the corresponding stars detected; otherwise, the stars detected cannot be identified.

\subsubsection{Flowchart of our method}

For a more straightforward illustration of the proposed star identification method, we constructed a flowchart of our algorithm, illustrated in Fig. 4.

\section{Simulation and Analysis}

Several simulations have been conducted to evaluate the star identification method proposed in this paper. Successful identification of a star image needs to satisfy the following two conditions ${ }^{16)}$ : (1) At least two true stars are correctly identified; and (2) there should be neither false stars identified nor true stars identified incorrectly. To better analyze 


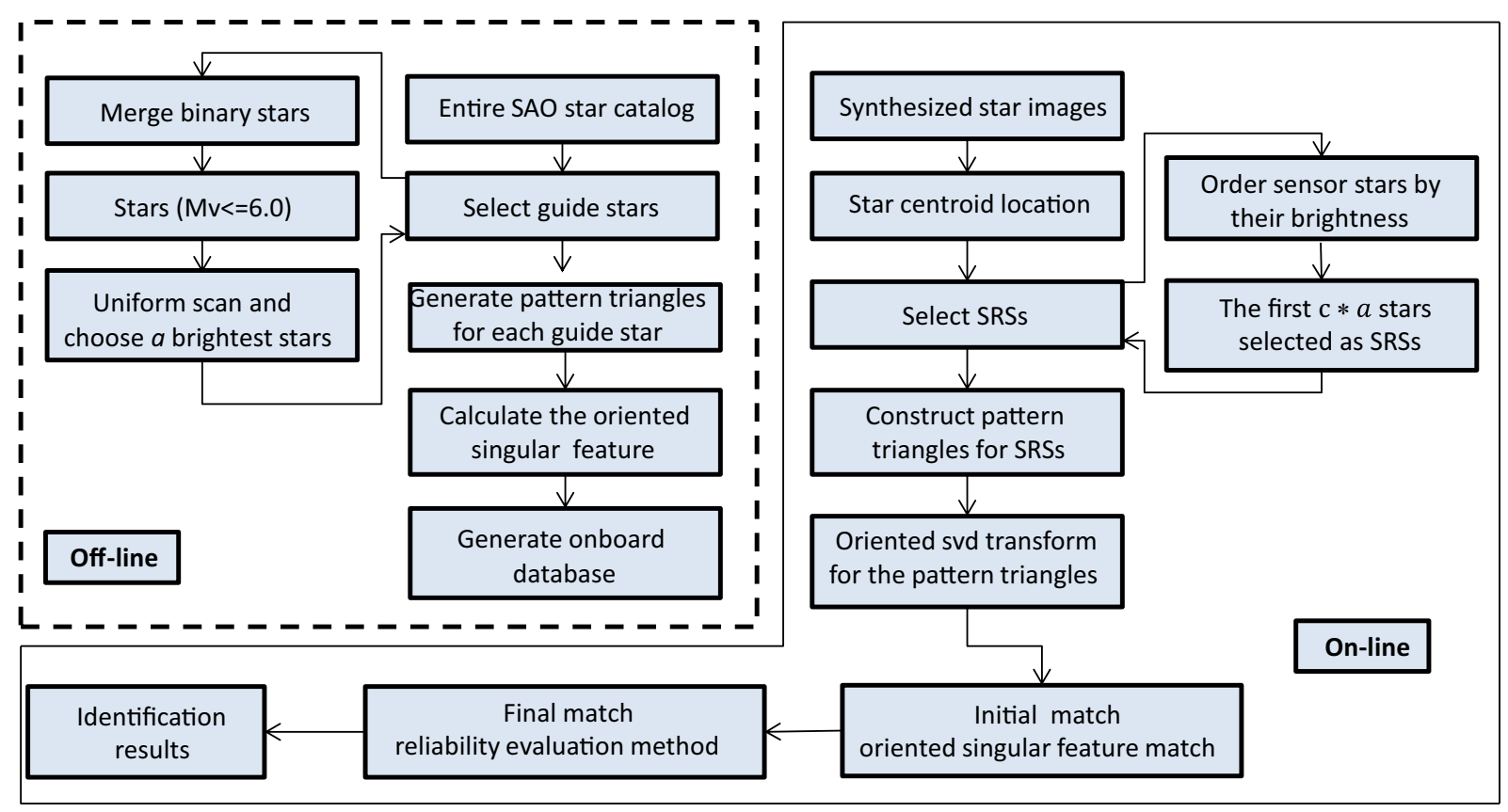

Fig. 4. Flowchart of our algorithm.

Table 1. Key parameters of the algorithm proposed.

\begin{tabular}{ll}
\hline Buffer radius $(r)$ & $0.3^{\circ}$ \\
Pattern radius $(R)$ & $5.0^{\circ}$ \\
$p_{1}$ match error $\left(\varepsilon_{1}\right)$ & 0.02 \\
$p_{2}$ match error $\left(\varepsilon_{2}\right)$ & 0.02 \\
\hline
\end{tabular}

the characteristics of the algorithm proposed, it is compared with the grid algorithm and SVD algorithm under various levels of star position noise and magnitude noise. We chose the SVD algorithm because a similar SVD method is utilized in our algorithm, and the grid algorithm, because it exhibits excellent performance in space missions.

All algorithms are programmed in MATLAB R2015a and carried out on a computer with an Intel Core $3.4 \mathrm{GHz}$ processor and Windows 7 environment.

\subsection{Parameters in simulation}

In simulations, the star sensor configuration is assumed to be $16 \times 16 \mathrm{deg}$ FOV with an image plane consisting of $1024 \times 1024$ pixels, and the pixel size is $0.015 \mathrm{~mm} \times$ $0.015 \mathrm{~mm}$. The principle point of the image is $(512,512)$. The focal length of the optical system is $54.646 \mathrm{~mm}$. The minimum sensitivity of star magnitude is set to $6.0 \mathrm{Mv}$.

The SAO J2000 star catalog, which contains 9040 stars with apparent magnitudes ranging down to 6.5 , is used to synthesize star images for the simulation.

The key parameters of the algorithm proposed are listed in Table 1.

The parameters $\varepsilon_{1}$ and $\varepsilon_{2}$ used in the initial matching step are important to correctly identify the image obtained. When tolerances $\varepsilon_{1}$ and $\varepsilon_{2}$ are at small values, correct candidates will be eliminated and the number of stars identified by the algorithm reduced. However, if tolerances $\varepsilon_{1}$ and $\varepsilon_{2}$ are large values, more redundant candidates may emerge in the initial step. As a result, there will be more candidate stars in the re-

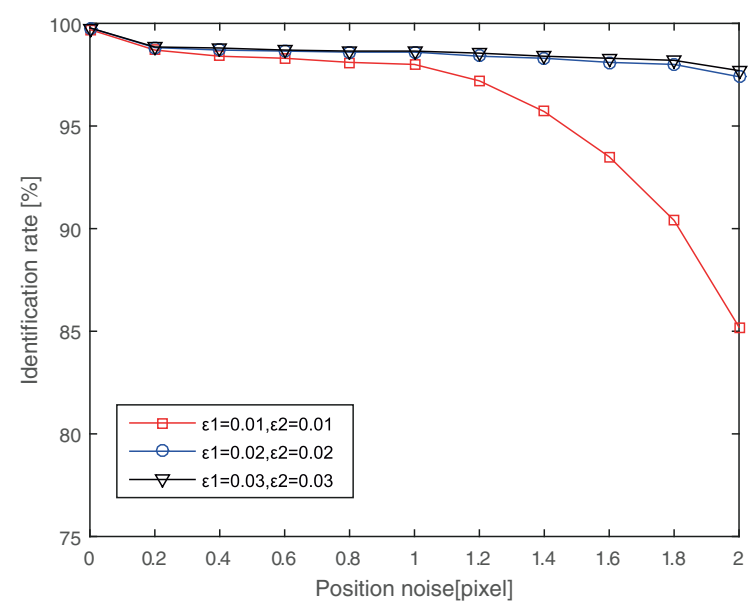

Fig. 5. Identification rate versus positional noise

liability evaluation, which requires more time to find unique matching results.

Figure 5 shows how the identification rates change when the standard deviation of position noise increases from 0 to 2.0 pixels. It can be seen that the identification rate drops quickly as the position noise increase above 1 pixels when $\varepsilon_{1}, \varepsilon_{2}$ is $0.01,0.01$, while the identification rates are maintained at a higher level in two cases where $\varepsilon_{1}, \varepsilon_{2}$ is set to $0.02,0.02$ and $0.03,0.03$. However, when $\varepsilon_{1}, \varepsilon_{2}$ is 0.02 , 0.02 , the execution time is $35.2 \mathrm{~ms}$, while it increases to $39.3 \mathrm{~ms}$ when $\varepsilon_{1}, \varepsilon_{2}$ is $0.03,0.03$. Considering both robustness and time performance, $\varepsilon_{1}, \varepsilon_{2}$ is set to $0.02,0.02$ in the following simulations.

\subsection{Comparison and analyses}

The impact of star position noise, magnitude noise and false stars in the FOV on the performance of three different algorithms is mainly estimated from two measurements in 


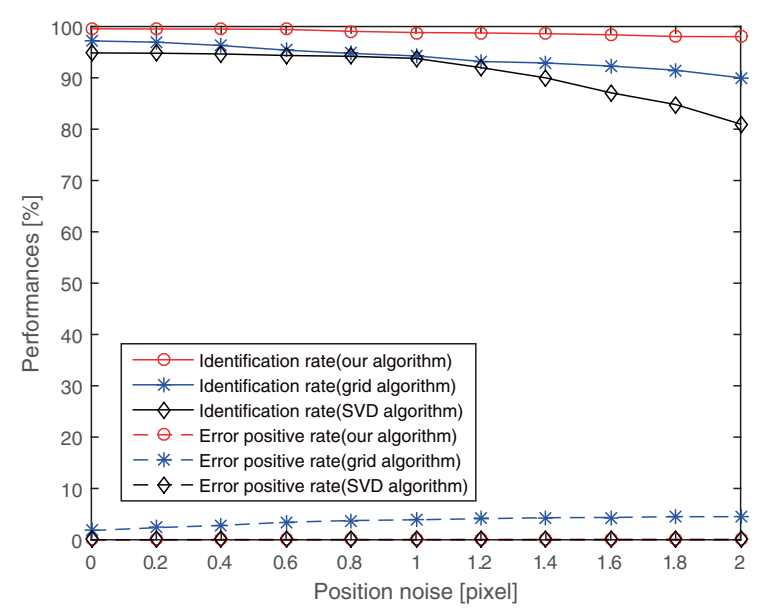

Fig. 6. Performance versus positional noise.

this section. One is the identification rate, the ratio of the number of star images that are successfully identified to the total number of star images in the experiment. The other is the error positive rate, the ratio of the number of star images misidentified to the number of simulations. The error positive is also important for star sensors. Even if an identification fails, the subsequent identifications follow in a continue manner. However, an error positive will supply a false attitude reference data.

In the presented simulation, one noise source is maintained at a typical level while the other varies linearly. For each condition, 10000 simulations are performed with the Monte-Carlo method to obtain the identification rate and the error positive rate of each algorithm. The confidence level of results in this section is $97.5 \%$, the error range is $\pm 0.04 \%$.

Optical distortion of the sensor lens, quantization error and the sub-pixel centroid algorithm used to extract star locations cause some star positional noise. In the simulation, by adding a random Gaussian noise to the ideal centroid locations of detected stars, the position noise is included in the synthesized star images. The brightness of stars cannot be measured accurately due to multiple reasons, so a random Gaussian noise is added to brightness of stars as well. The magnitude noise allows some dimmer stars whose brightness falls below the sensitivity of the star sensor to actually appear and stars brighter than the instrumental threshold to be lost in the synthesized star images. Moreover, false stars may also appear in the image.

(1) Robustness toward star positional noise: Figure 6 shows the identification and the error positive rates for the grid, SVD, and proposed algorithms as the standard deviation of the position noise increases from 0.0 to 2.0 pixels, while the standard deviation of magnitude noise remains at $0.3 \mathrm{Mv}$.

As shown in Fig. 6, our algorithm has superior performance compared to the grid and SVD algorithms considering robustness to position noise. The identification rate of the algorithm proposed drops slightly, from $99.6 \%$ to $97.0 \%$, as the position noise added to the star image increases from 0 to 2.0 pixels. In contrast, the rate of the grid algorithm decreases

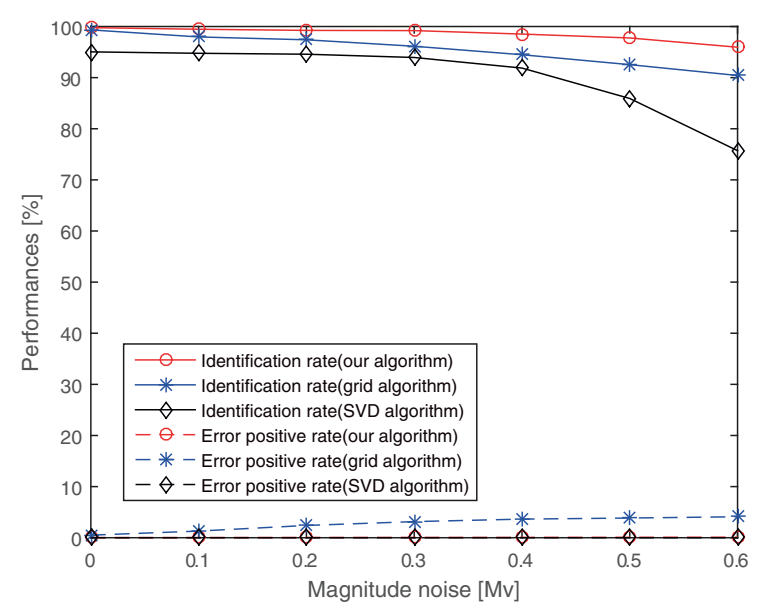

Fig. 7. Performance versus magnitude noise.

from $97.2 \%$ to $90.0 \%$, and performance of the SVD algorithm is poorer, dropping quickly from $95.0 \%$ to $81.1 \%$. In terms of the error positive rate, the proposed and SVD algorithms obtain lower error rates with position noise, while the error positive rate of the grid algorithm increases from $1.8 \%$ to $4.5 \%$. This is due to position noise causing the wrong closest neighboring star to be chosen in the process of constructing the grid pattern.

(2) Robustness toward star magnitude noise: Figure 7 shows how identification rates change for the grid, SVD, and proposed algorithms as the standard deviation of the magnitude noise increases from 0 to $0.6 \mathrm{Mv}$, while the standard deviation of the position noise is maintained at 0.5 pixels.

It can be seen that the algorithm proposed is the most robust among the three algorithms considering magnitude noise. The identification rate of our algorithm maintains a level of over $96.2 \%$ as the standard deviation of star brightness error increases from 0 to $0.6 \mathrm{Mv}$. In contrast, the identification rate of the grid algorithm drops quickly from $99.3 \%$ to $90.4 \%$ because generating a grid pattern requires selection of the closest neighboring star, which is sensitive to uncertain magnitude noise. The SVD algorithm rate decreases from $95.0 \%$ to $75.7 \%$ because this algorithm selects the four brightest stars in the image to form a pattern, which is easily influenced by brightness noise. Furthermore, the error positive rate of the algorithm proposed is similar to that of the SVD algorithm when large magnitude noise is added to the image, while the grid algorithm once more falls behind in performance of the error positive rate.

(3) Robustness toward false stars: Figure 8 shows identification rates of the grid, SVD and proposed algorithms considering the number of false stars. The star images acquired by the sensor contain several false stars as a result of hot spots, planets, or radiation. The standard deviation of star position noise is fixed at 0.5 pixels, and the magnitude error is $0.3 \mathrm{Mv}$. In the simulation, we scatter from 0 to 3 false stars, each with a random brightness ranging from $4.0 \mathrm{Mv}$ to 6.0 Mv.

When there are no false stars present in the image, our algorithm, the grid and SVD algorithm achieve identification 


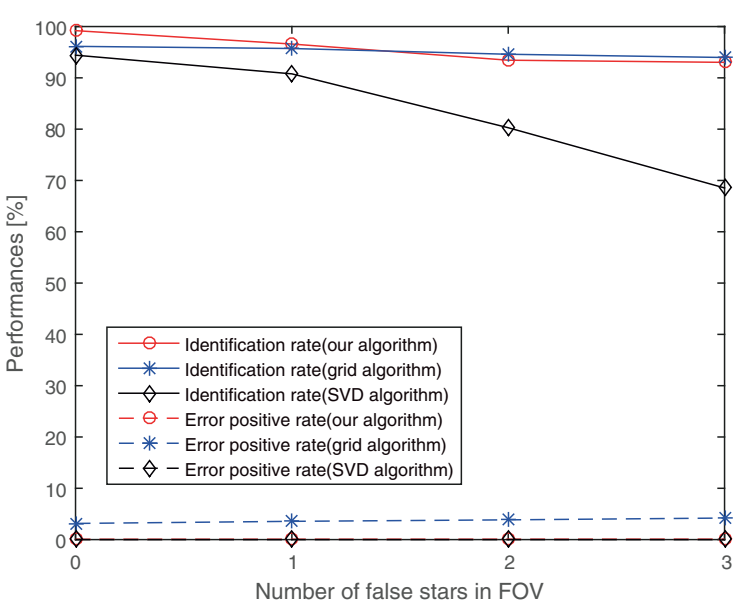

Fig. 8. Performance versus false stars.

Table 2. Time and memory cost.

\begin{tabular}{lccr}
\hline Algorithm & Our method & Grid & \multicolumn{1}{c}{ SVD } \\
\hline Average time & $35.2 \mathrm{~ms}$ & $185.6 \mathrm{~ms}$ & $13.4 \mathrm{~ms}$ \\
Maximum time & $56.3 \mathrm{~ms}$ & $228.1 \mathrm{~ms}$ & $21.9 \mathrm{~ms}$ \\
Memory cost & $145.0 \mathrm{~KB}$ & $331.0 \mathrm{~KB}$ & $1483.0 \mathrm{~KB}$ \\
\hline
\end{tabular}

rates of $99.2 \%, 96.1 \%$, and $94.4 \%$, respectively. As the number of false stars increases, the identification rate of the SVD algorithm decreases quickly, while the rates of the other two algorithms remain relatively stable. In many failed cases, the SVD algorithm mistakenly chooses a false star to construct the pattern. Our algorithm and the grid algorithm obtain identification rates of $96.6 \%$ and $95.7 \%$, respectively, when only one false star is added to the image. As the number of false stars increases to three, the identification rate of the grid algorithm maintains a level of over $93.8 \%$, while that of the algorithm proposed drops to $93.2 \%$, which is slightly lower than that of the grid algorithm. However, the error positive rate of the grid algorithm is higher than that of our method. This is because some false stars may be identified as the closest star, resulting in false identification by the grid algorithm.

(4) Memory and time performance: The memory cost and identification time for the three algorithms are shown in Table 2. The memory cost is the total memory size of the onboard database that consists of the reference catalog and pattern database of the algorithm. Our algorithm has been proven to require less memory than both the grid algorithm and SVD algorithm.

The average time in Table 2 refers to the average time it takes for the algorithm to identify a star image; that is, the average time interval between inputting the measurement information (i.e., the coordinates and apparent brightness of detected stars) and the output of the final matching results. The absolute identification times in Table 2 cannot be directly compared with those of actual star sensors, but it is nevertheless feasible to evaluate the relative time performance of the different algorithms. The average identification time of the algorithm proposed is $35.2 \mathrm{~ms}$, which is slightly
Table 3. Percentages of $\mathrm{H}, \mathrm{M}$ and $\mathrm{L}$ images versus magnitude noise.

\begin{tabular}{ccccc}
\hline Magnitude noise & $\mathrm{H}$ & $\mathrm{M}$ & $\mathrm{L}$ & Others \\
\hline $0.0 \mathrm{Mv}$ & $92.4 \%$ & $6.9 \%$ & $0.5 \%$ & $0.2 \%$ \\
$0.1 \mathrm{Mv}$ & $91.6 \%$ & $7.1 \%$ & $0.7 \%$ & $0.6 \%$ \\
$0.2 \mathrm{Mv}$ & $91.3 \%$ & $7.2 \%$ & $0.8 \%$ & $0.7 \%$ \\
$0.3 \mathrm{Mv}$ & $90.4 \%$ & $7.6 \%$ & $1.2 \%$ & $0.8 \%$ \\
$0.4 \mathrm{Mv}$ & $89.1 \%$ & $8.1 \%$ & $1.4 \%$ & $1.4 \%$ \\
$0.5 \mathrm{Mv}$ & $87.2 \%$ & $8.5 \%$ & $1.7 \%$ & $2.6 \%$ \\
$0.6 \mathrm{Mv}$ & $84.6 \%$ & $9.3 \%$ & $1.9 \%$ & $3.8 \%$ \\
\hline
\end{tabular}

slower than the SVD algorithm $(13.4 \mathrm{~ms})$, but much faster than the grid algorithm (185.6 ms), which utilizes lookup table technology to search for candidates. Moreover, the maximum identification time of the algorithm proposed is $56.3 \mathrm{~ms}$, which is also acceptable for practical applications.

Considering the acceptable memory requirements and identification time consumption, Table 2 demonstrates that our algorithm performs better than the grid and SVD algorithms.

\subsection{Reliability level ratio}

In this section, we discuss the variation in the percentages of star images labeled with high (H), medium (M), and low (L) reliability levels when using the algorithm proposed considering the magnitude noise level and number of false stars in the FOV. The results of simulations are briefly analyzed.

Table 3 shows the percentages of star images labeled as high $(\mathrm{H})$, medium $(\mathrm{M})$, and low $(\mathrm{L})$ reliability levels as the standard deviation of the magnitude noise increases from 0.0 to $0.6 \mathrm{Mv}$, while the standard deviation of position noise remains at 0.5 pixels. It can be seen that the percentage of $\mathrm{H}$ level images drops from $92.4 \%$ to $84.6 \%$ as the magnitude noise increases from 0 to $0.6 \mathrm{Mv}$, while the percentages of M-level and L-level images increase from $6.9 \%$ and $0.5 \%$ to $9.3 \%$ and $1.9 \%$, respectively. The algorithm proposed selects two of the brightest neighboring stars in the process of generating triangle patterns. However, the wrong neighboring stars may be selected due to magnitude noise, which causes a reduction in the correct candidates involved in the voting process. Therefore, the percentage of H-level images decreases, while the percentage of M-level and L-level images increases when the magnitude noise is added to star images.

Table 4 shows the percentage of star images labeled as high $(\mathrm{H})$, medium $(\mathrm{M})$, and low $(\mathrm{L})$ reliability level as the number of false stars in the FOV increases from 0 to 3 , while the position noise is 0.5 pixels and the magnitude noise is $0.3 \mathrm{Mv}$. As the number of false stars increases from 0 to 3, we observe that the percentage of H-level images drops quickly from $90.4 \%$ to $69.3 \%$, while the percentage of M-level images increases from $7.6 \%$ to $17.5 \%$, and the percentage of L-level images increases from $1.2 \%$ to $6.3 \%$. False stars may be selected as the brightest neighboring stars to construct triangle patterns, which decreases the number of correct candidates obtained in the initial matching step. As a result, the percentage of $\mathrm{H}$-level images decreases rapidly, while the percentages of M-level and L-level images increase as the number of false stars in the image increases. 
Table 4. Percentages of $\mathrm{H}, \mathrm{M}$ and $\mathrm{L}$ images versus false stars.

\begin{tabular}{ccrcc}
\hline False stars & $\mathrm{H}$ & $\mathrm{M}$ & $\mathrm{L}$ & Others \\
\hline 0 & $90.4 \%$ & $7.6 \%$ & $1.2 \%$ & $0.8 \%$ \\
1 & $82.2 \%$ & $10.9 \%$ & $3.5 \%$ & $3.4 \%$ \\
2 & $75.7 \%$ & $13.8 \%$ & $3.9 \%$ & $6.6 \%$ \\
3 & $69.3 \%$ & $17.5 \%$ & $6.3 \%$ & $6.9 \%$ \\
\hline
\end{tabular}

\subsection{Failure example analysis}

Figure 9 shows two examples of failure in simulations using the algorithm proposed. There are 21 stars detected in Fig. 9(a), among which \#20 and \#21 are false stars. Some SRSs are located at the edge of the image, and thus the number of neighboring stars in the pattern radius is less than 2, which increases the likelihood that the correct two brightest neighboring stars are outside the field of view, and false stars \#20 and \#21 are selected to form some triangle patterns due to their high brightness. For the above-mentioned reasons, the number of triangle patterns correctly constructed in Fig. 9(a) is 0, and as a consequence, our method fails in star identification. In Fig. 9(b), 11 stars are detected by the sensor, but only one triangle pattern is correctly constructed (i.e., denoted by the red triangle). However, SRS triangle patterns are usually similar to several triangle patterns in the database, which leads to ambiguous matching. The post-processing of "star voting" cannot obtain unique matching results in cases where there is only one correct candidate after the initial match. Therefore, the star image in Fig. 9(b) is not successfully identified by the algorithm proposed.

\section{Mathematical Model}

In this section, we employ the probabilistic model to analyze the performance of the algorithm proposed, and it comprehensively explains the reason why our algorithm is robust and efficient for star identification.

In the initial algorithm matching process, oriented singular feature matching is performed to obtain candidate stars. This essentially searches for triangle patterns in the database that are similar to those in the sensor. According to the analytical approach regarding star identification reliability reported in Kumar et al., ${ }^{17)}$ when there is an uncertainty $\sigma$ in the angular distance measurement, the expected number of candidates that match the given star triangle detected $\triangle i j k$ is expressed as follows

$$
\begin{aligned}
E_{i j k}= & \frac{N(N-1)(N-2)}{8 \pi} \\
& \times\left(\theta_{i}^{+}+\theta_{i}^{-}\right)\left(\theta_{j}^{+}+\theta_{j}^{-}\right) \sin (\sigma) \sin \left(\theta_{i j}\right) \sin \left(\theta_{i k}\right) \sin \left(\theta_{j k}\right)
\end{aligned}
$$

where, $N$ denotes the total number of stars with a magnitude less than the minimum sensitivity of the sensor, $\sigma$ is the measurement error, and $\theta_{i j}, \theta_{i k}$, and $\theta_{j k}$ are the corresponding three angular distances of the triangle. We have the following expressions for $\theta_{i}^{+}$and $\theta_{i}^{-}$in Eq. (7), and similar expressions can be obtained for $\theta_{j}^{+}$and $\theta_{j}^{-}$.

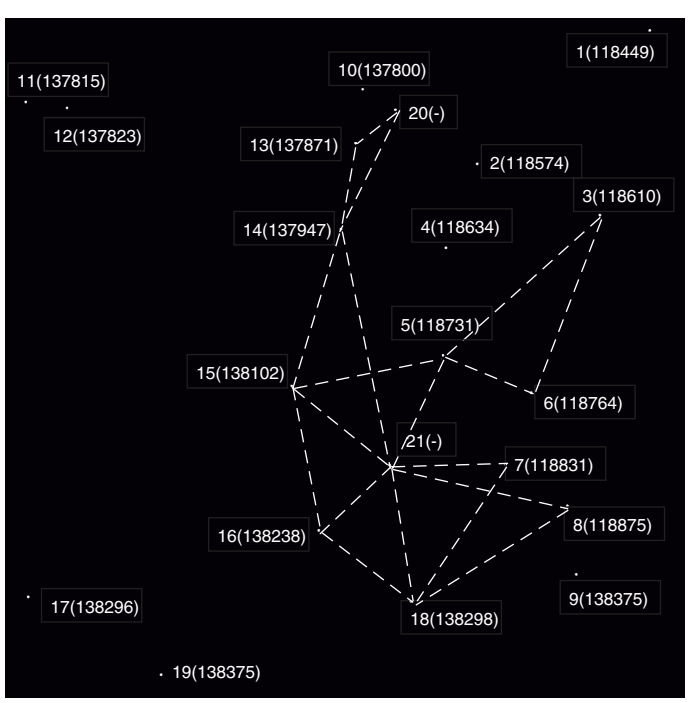

(a) No Correct constructed patterns.

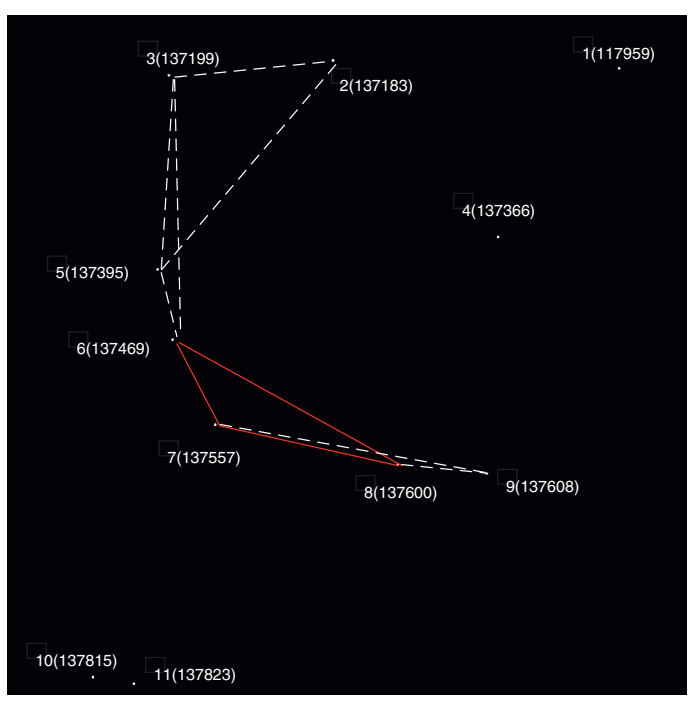

(b) One correct constructed pattern.

Fig. 9. Failure examples.

$$
\begin{gathered}
\theta_{i k}^{+}=\arccos \left(\cos \sigma \cos \theta_{i k}+\sin \sigma \sin \theta_{i k} \cos \theta_{k}\right) \\
\theta_{i k}^{-}=\arccos \left(\cos \sigma \cos \theta_{i k}-\sin \sigma \sin \theta_{i k} \cos \theta_{k}\right) \\
\theta_{i}^{+}=\arcsin \left(\frac{\sin \sigma \sin \theta_{k}}{\sin \theta_{i k}^{+}}\right) \\
\theta_{i}^{-}=\arcsin \left(\frac{\sin \sigma \sin \theta_{k}}{\sin \theta_{i k}^{-}}\right)
\end{gathered}
$$

Considering the configuration of the star sensor in this paper, the average expectation of the number of candidates is theoretically 5.4 with a pixel noise of 0.5 pixels, while the statistical results in the simulation test of our method yields 2.32 candidates. This is mainly because the oriented singular feature performs better in anti-noise conditions and the oriented coefficient eliminates the possibility of mismatching the mirror triangle patterns.

In terms of search efficiency, our algorithm employs a fast binary search method to select catalog stars from the database that have corresponding oriented singular values lo- 
cated within a certain interval. The time complexity of the method proposed is $\mathrm{O}(\log N)$, which is much lower than $\mathrm{O}(N)$ of the traversal search method. Therefore, our method requires less time to find candidates in the matching process.

We assume the entire celestial sphere with a uniform star distribution, and given a detected star pair, such as stars $i$ and $j$, and the angular distance between them is denoted by $\theta_{i j}$. Therefore, the number of candidates expected to match the given observed star pair with uncertainty is easily obtained using the following equation:

$$
E_{i j}=\frac{N(N-1)}{2} \sin \sigma \sin \theta_{i j}
$$

In the star voting process, given the number of stars detected by the sensor $M$, the number of candidate stars expected whose corresponding counter values are $M-1$ for the star observed $i$ is expressed as follows

$E(M-1, M-1)=N[(N-1) \sin \sigma]^{M-1} \prod_{j=j_{1}}^{j_{M-1}} \sin \theta_{i j}$

Considering the typical values: $N=5000$ and $\sigma=$ $160 \mu \mathrm{rad}$, the theoretical estimates of the number of candidate stars expected for $M=3,4$ and 5 are shown in Table 5 .

Some star pairs participating in the voting may practically lead to an incorrect match, which reduces the reliability of identification results to a certain extent. Generally, there are several ways to make this scenario occur.

First, spurious non-star items, such as space debris, radiation, and hot spots, are contained in the images acquired, and the star sensor might recognize them as stars. Second, some stars that can actually be detected by the image plane obtained are not included in the database. A third cause is due to large measurement error in angular distance. Therefore, correct matches of these pairs are always excluded from the candidates in the star voting process. We define these pairs as invalid voting pairs, whereas the rest are valid voting pairs. Provided that there are only $H$ valid voting pairs among all $M-1$ star pairs associated with the star detected $i$, the overall number of candidate stars with $H$ votes is approximated as follows:

$$
E(M-1, H) \approx N[(N-1) \sin \sigma]^{M-1} \sum_{m=1}^{C(M-1, H)} \prod_{j=1}^{H} \sin \theta_{i j_{m}}
$$

where, $C(M-1, H)$ combinations exist with $H$ randomly selected elements from the $M-1$ star pairs, with $j_{m}$ denoting the $j$ th star in the $m$ th combination.

We can draw several conclusions from Eq. (13) and Eq. (14). In star voting, the number of candidates expected for the star detected $i$ is determined by several factors, including the overall number of visible stars $N$, measurement error $\sigma$, number of stars imaged in the sensor plane $M$, number of valid voting pairs $H$, and the angular distance from star $i$ to other stars detected $\theta_{i j}$.

The stars detected having a high corresponding number of expected candidates $E$ have a higher frequency of possible mismatches. Our method employs a number of strategies to
Table 5. Theoretical estimate results

\begin{tabular}{cc}
\hline$M-1$ & Theoretical expectation \\
\hline 2 & 304 \\
3 & 67 \\
4 & 11 \\
\hline
\end{tabular}

decrease $E$. First, the guide stars involved in the voting process are filtered by oriented singular feature matching, such that $N$ is significantly reduced from the number of all catalog stars of the entire celestial sphere to the number of candidate stars. Second, the stars detected with candidates are preserved after the initial matching. Therefore, these stars are probably guide stars in the onboard database, which avoids invalid voting pairs caused by non-star items or visible stars not included in the catalog as much as possible. As a result, $H$ is almost equal to $M-1$, and the statistical results in the simulation test show that the expected values of $H$ and $M-1$ are 7.45 and 7.82 , respectively, when 0.5 -pixel position noise and $0.3-\mathrm{Mv}$ magnitude noise are added to the image. Finally, the stars observed participating in the reliability evaluation step have high brightness, and the coordinates of brighter stars can generally be determined with higher precision.

\section{Conclusions}

This paper has describes a full-sky autonomous star identification algorithm that is intended to solve the "lost-inspace" problem for spacecraft attitude determination. The reliable oriented singular values of stars detected are used to obtain the initial candidate stars in the database, and the follow-up reliability evaluation process generates the final matching results. Simulation results show that the identification rate of the algorithm proposed is higher than that of the grid and SVD algorithms when considering the different levels of star position noise and magnitude noise, and also exhibits good robustness to false stars in the FOV. Moreover, the memory required for our algorithm is smaller than that of the other two algorithms, and the calculation time is reasonable, making it suitable for actual astronautic missions. Future work will focus on incorporating our algorithm into a real star sensor to validate on-orbit performance.

\section{Acknowledgments}

This work is supported by the 863 program No. 2015AA7046612.

\section{References}

1) Junkins, J. L., White, C. C. I., and Turner, J. D.: Star Pattern Recognition for Real Time Attitude Determination, J. Astronaut. Sci., 25, 25 (1977), pp. 251-270.

2) Wang, J., Cho, S., and Chun, J.: Initial Attitude Determination Using a Single Star Sensor, Trans. Jpn. Soc. Aeronaut. Space Sci, 50 (2007), pp. 27-33.

3) Spratling, B. B. and Mortari, D.: A Survey on Star Identification Algo- 
Trans. Japan Soc. Aero. Space Sci., Vol. 62, No. 5, 2019

rithms, Algorithms, 2, 1 (2009), pp. 93-107.

4) Padgett, C. and Kreutz-Delgado, K.: A Grid Algorithm for Autonomous Star Identification, IEEE Trans. Aerospace Electronic Syst., 33, 1 (1997), pp. 202-213.

5) Na, M., Zheng, D., and Jia, P.: Modified Grid Algorithm for Noisy AllSky Autonomous Star Identification, IEEE Trans. Aerospace Electronic Syst., 45, 2 (2009), pp. 516-522.

6) Clouse, D. S. and Padgett, C. W.: Small Field-of-view Star Identification Using Bayesian Decision Theory, IEEE Trans. Aerospace Electronic Syst., 36, 3 (2000), pp. 773-783.

7) Lee, H. and Bang, H.: Star Pattern Identification Technique by Modified Grid Algorithm, IEEE Trans. Aerospace Electronic Syst., 43, 3 (2007), pp. 1112-1116.

8) Silani, E. and Lovera, M.: Star Identification Algorithms: Novel Approach \& Comparison Study, IEEE Trans. Aerospace Electronic Syst., 42, 4 (2006), pp. 1275-1288.

9) Zhang, G., Wei, X., and Jiang, J.: Full-sky Autonomous Star Identification Based on Radial and Cyclic Features of Star Pattern, Image Vision Comput., 26, 7 (2008), pp. 891-897.

10) Wei, X., Zhang, G., and Jiang, J.: Star Identification Algorithm Based on Log-Polar Transform, Optical Technique, 6, 8 (2006), pp. 1-31.

11) Juang, J. N., Kim, H. Y., and Junkins, J. L.: An Efficient and Robust
Singular Value Method for Star Pattern Recognition and Attitude Determination, Adv. Astronaut. Sci., 115, 1 (2003), pp. 211-220.

12) Liebe, C. C.: Star Trackers for Attitude Determination, IEEE Aerospace Electronic Syst. Magazine, 10, 6 (1995), pp. 10-16.

13) Zhang, G. J., Wei, X. G., and Jiang, J.: Star Map Identification Based on a Modified Triangle Algorithm, Acta Aeronautica Astron. Sinica, 27, 6 (2006), pp. 1150-1154.

14) Mortari, D., Samaan,, M. A., Bruccoleri, C., and Junkins, J. L.: The Pyramid Star Identification Technique, Navigation, 51, 3 (2004), pp. 171-183.

15) Van Bezooijen, R.: Techniques for Optimizing an Autonomous Star Tracker, U.S. Patent 5745869, 1998.

16) Wang, G., Li, J., and Wei, X.: Star Identification Based on Hash Map, IEEE Sensors J., 18, 4 (2018), pp. 1591-1599.

17) Kumar, M., Mortari, D., and Junkins, J. L.: An Analytical Approach to Star Identification Reliability, Acta Astronautica, 66, 3 (2013), pp. $508-515$.

Saburo Matunaga Associate Editor 